\title{
Developing culturally competent and compassionate healthcare leaders: A European model
}

\author{
Christiana Kouta*1, Elena Rousou ${ }^{1}$, Vasiliou Marios ${ }^{1}$, Irena Papadopoulos ${ }^{2}$ \\ ${ }^{1}$ Cyprus University of Technology, Cyprus \\ ${ }^{2}$ Middlesex University, UK
}

Received: January 13, 2019

Accepted: March 24, 2019

Online Published: April 15, 2019

DOI: $10.5430 /$ jnep.v9n7p109

URL: https://doi.org/10.5430/jnep.v9n7p109

\begin{abstract}
Objective: This paper aims to describe the development of a European model that refers for healthcare leadership. The model promotes the values of cultural competence and compassion.

Methods: The development of this model is part of the IENE $4 \mathrm{EU}$ funded project with participating countries: United Kingdom, Spain, Cyprus, Romania, Italy, Denmark, Turkey. Its development is based on a) a needs assessment survey among healthcare leaders in the partner countries $(\mathrm{N}=199)$, b) two round Delphi study with 14 experts and c) a focus group with healthcare leaders after the development of the model.

Results: The components of this model include the basic principles, values and skills that a health care leader should have as a role model and a coach of his/her staff in delivering compassionate and culturally competent care. This model was further used within the IENE 4 project, as a tool for creating learning tools, aiming to improve the quality of care within a cultural framework. Fourteen such learning tools were developed and piloted in all partner countries.

Conclusions: Health care leaders need to guide, mentor and support their staff and collaborate among them and with patients and families, as to provide quality care within a safe, compassionate and culturally appropriate environment. This model highlights the key principles of culturally competent and compassionate health care leadership.
\end{abstract}

Key Words: Health care leaders, Cultural competence, Compassionate health care, Leadership

\section{INTRODUCTION}

In contemporary years in health care, many challenges are apparent, such as population diversity, migrants, refugees, new diseases, re-occurrence of old diseases and so on and prioritization of required competences and behaviour has begun to shift. ${ }^{[1]}$ Effective healthcare leadership improves major clinical outcomes in patients as well as health professionals' well-being. ${ }^{[2]}$

Literature $^{[3,4]}$ suggest that two of the future (and current) challenges for healthcare are those related to diversity and compassion. It is incumbent upon those who hold formal leadership positions, but also everyone within healthcare systems to address these two challenges, to equip themselves with the relevant competencies and to create and nurture the working conditions to inspire others to follow their lead. Leadership models are helpful for learners to grasp new concepts, understand lessons learned through their experiences, and facilitates comprehension through reflection. ${ }^{[2]}$ Further, effective healthcare leadership is a skill that must be and can be taught, ${ }^{[2]}$ taking in consideration today's cultural competence demands.

\footnotetext{
*Correspondence: Christiana Kouta; Email: christiana.kouta@cut.ac.cy; Address: Department of Nursing, Cyprus University of Technology 15 Vragadinou str., Limassol, Cyprus.
} 
Health care leaders, both at service planning and service delivery levels, need to have an understanding of the perspectives, traditions, values, practices, and family systems of culturally diverse individuals, families, communities and populations for whom they care, as well as knowledge of the complex variables that affect the achievement of health and wellbeing. Understanding the process for assessing cultural patterns and factors that influence individual and group differences is critical in the development and delivery of culturally appropriate and compassionate services.

Healthcare systems are also complex and diverse in terms of the numerous professional groups, departments and specialities with intricate, non-linear interactions between them. Within healthcare systems, the different professional groups diverse subcultures might support or conflict with each other. The result of such constraints are the difficulties in delivering safe, appropriate and compassionate care.

Leaders need to have a vision that is well communicated and ability in building trust among colleagues, both of which are motivating factors to inspire staff whilst instilling the passion and energy needed to move towards the common goals. ${ }^{[5,6]}$

Compassion in nursing and other health care professions, is a taken-for-granted notion, and that nurses are naturally compassionate, or they know what compassion is and how to demonstrate it, are notions which need to be challenged. ${ }^{[7]}$ Compassionate care leads to higher levels of patient/family satisfaction, which in turn contribute to faster more effective healing and higher levels of compliance acquiescence, the consequence of which is better use of resources and even lower costs. ${ }^{[8]}$ In addition, compassionate leaders make the best role models for nurses in practice, which in turn become compassionate role models to students. ${ }^{[9]}$ The evidence provided in this brief section supports that leadership in healthcare settings should strive to be culturally competent and compassionate.

The added value of this study is that it goes beyond usual leadership health care models and focuses on cultural competence and compassionate care as these are considered essential components of quality and effective holistic care. This article discusses the development of a European model for healthcare leadership that promotes the values of cultural competence and compassion.

\section{The IENE 4 project}

The IENE 4 (Intercultural Education for Nurses in Europe) project was funded by the EU ERASMUS+ programme. One of the objectives of the project was to produce a leadership model that could respond to the leadership challenges, as outlined above, to enhance, empower and develop culturally competent and compassionate leadership. Seven European countries participate as partners in IENE 4 project: UK (coordinator), Spain, Cyprus, Romania, Italy, Denmark and Turkey.

An integrative review was applied in three thematic areas: Universal components of compassion; Measuring culturally competent compassion; Learning culturally competent compassion in theory and practice. All literature was assessed against the inclusion criteria, and the CASP (Critical Appraisal Skills Programme) checklist was applied for cohort studies as a quality assessment tool. In the thematic area of universal components of compassion, 25 studies were included (2000-2014); in measuring culturally competent compassion, 6 studies were included (1999-2013) and in learning culturally competent compassion, 16 studies were included (2001-2014). Findings revealed that compassion is a quality care dimension and providing culturally competent care can be learned. To achieve an effective learning and training of culturally competent and compassionate care within healthcare context, health care professionals require appropriated support and strong leadership. ${ }^{[10]}$

Further, through the integrative literature review, the following definition of Culturally Competent and Compassionate healthcare Leadership (CCCL) was derived: "CCCL is the process that a leader goes through in demonstrating culturally aware, knowledgeable, sensitive, competent and compassionate standards of leadership and care. S/He adopts and applies leading principles and values, and moral virtues; inspires others with his/her example and vision; provides quality, appropriate and equal health care; becomes a role model and acts within a culturally competent and compassionate working environment that s/he develops and guides". ${ }^{[1]}$

\section{Methods}

The main research questions used as a basis for the development of the model:

- What are the basic elements needed for a culturally competent and compassionate leadership?

- Which values, philosophy and educational principles should be included in a CCCL model?

A mixed research approach was used to answer these questions. First, an integrative review of literature was performed pertaining the universal components of compassion as well as the measurement, theory and practice of compassion. Based on the results, a questionnaire was developed, and a needs assessment survey was conducted among healthcare leaders in all partner countries. Additionally, relevant statements were developed, which formed the basis of a Delphi study aiming for a consensus of common statements and principles. The 
Delphi method is a popular tool for identifying and prioritizing issues for managerial and leadership decision-making. ${ }^{[12]}$

Once the model was developed, it was discussed in a focus group with health care leaders. Thus, the model was finalized.

\subsection{Needs assessment survey}

The purpose of the survey was to identify the needs among healthcare leaders both at management and senior front-line staff levels, which, if met, would enable them to become culturally competent and compassionate leaders.

\subsection{Tool}

A questionnaire was developed by the IENE 4 team, based on the integrative review. The review was based on the overall question: "How do nurses and other health professionals learn to practice culturally competent compassionate care?". Three searches were performed as described above: 1) which universal components of compassion are universal; 2) how can compassion in nurses and other health professionals be measured in multicultural clinical environments; 3 ) how do nurses and other health professionals learn to practice culturally competent compassionate care. ${ }^{[10]}$ From these reviews, information was extracted and formed the basis for the development of the needs assessment questionnaire. Standards identified in the aforementioned literature were also used as input in the Delphi rounds which followed. The use of the literature and experts is a common practice in helping to develop a questionnaire. ${ }^{[14]}$

The needs assessment questionnaire developed by the IENE 4 team, consisted of both open-ended and close-ended questions (values, knowledge, skills and qualities that a culturally competent and compassionate healthcare leader should have). ${ }^{[11]}$ Often questionnaires use both ways of asking questions (open/close ended) providing the chance to have a broad perspective of understanding what is under investigation. This is in line with health research tools or measurements. ${ }^{[14]}$ The close-ended questions included demographics (age, gender, job title, years of experience). Two questions were also included as for the participants to rank relevant statements, according to their importance: 1) In your opinion, which are the most significant elements of culturally competent and compassionate care in nursing? (rank the statements from 1-5) 2) In your view, what key leadership qualities are needed by senior healthcare staff in order to be able to promote a culturally competent and compassionate work culture? (rank the statements from 1-10). The open-ended question asked participants to state: key values, knowledge and skills needed for a healthcare leader as to enhance his/her ability to promote culturally competent and compassionate care in the areas and teams s/he is responsible for? The questionnaire was piloted with health care leaders $(\mathrm{N}=14)$ and reached to a consensus regarding the clarity of the statements and its relevance to the purpose of the study.

\subsection{Sample, sampling}

A convenience sample was used. Health professional leaders from various clinical settings (hospital and community) were approached via email through professional organizations and/or in person and informed about the purpose of the study in all partner countries. Inclusion criteria were: be nurse/health care leader, speak local language, wiliness to participate.

\subsection{Data collection}

The data was collected via the online administration of the questionnaire through Monkey survey platform. A link was sent via email to participants who met the inclusion criteria and voluntarily accepted to be involve in the study. Members of the IENE 4 team were in charge of the management data collection and the survey platform as to check for any missing answers and proceed with the analysis.

\subsection{Analysis}

Frequencies and percentages were reported for questions regarding personal information. In questions that items had to be ranked, mean rank given by responders was calculated. In the case of open-ended questions regarding the key values, knowledge and skills, researchers re-classified and refined answers with similarities to form a list of categories (values, skills, knowledge). The frequency by which each term was mentioned by participants was counted. The most frequent terms were selected by consensus to form the statements for the Delphi questionnaire.

\subsection{Delphi study}

Delphi methodology was used aiming for a consensus of common statements. The Delphi study was consisted of two rounds. It was decided to invite 14 international health care experts (2 from each partner country) to participate in the Delphi panel. The statements that were included in the Delphi study were based on the: 1) findings of three aforementioned literature reviews (as previously mentioned), which revealed that the key concepts measuring compassionate care included: empathy, recognizing and ending suffering, communication, patient involvement, competence and attending to patients' needs; 2) needs assessment survey, which revealed that the most frequently reported by the participants key values, knowledge and skills.

In Delphi study round 1, a total of 48 statements were developed and distributed accordingly in the four main stages 
based on the Papadopoulos Compassionate Care Model ${ }^{[13]}$ (Cultural Awareness $=15$, Cultural Knowledge $=19$, Cultural Sensitivity $=9$ and Cultural Competence $=5$ ). Further, experts $(\mathrm{N}=14)$, were asked to reflect on each statement's relevance to culturally competent and compassionate leadership and rate the statements on a 5-point scale on their perceived importance as: a) to which potential components should be included in a model of care delivery characterized by culturally competent compassionate leadership; b) on their clarity; and c) to state to which value, principle or philosophy specific to culturally competent compassion the statement applied. The panel members were encouraged to support their choices, to suggest alternatives or to suggest new items.

Each statement's mean score and ranking were measured. Statements which scored a mean above 4 and demonstrated consistently high median and modal rankings were retained (36 statements), as this was considered a consensus on a statement and the statement were perceived suitable for further development. Statements which scored a mean below 4 were omitted (12 statements).

In Delphi study round 2, a cross-check was performed of the retained statements with the needs analysis assessment (as for the values-principles that where thought as the most important by the participants). The final document of Delphi study round 2 included 41 statements (Cultural Awareness $=10$, Cultural Knowledge $=19$, Cultural Sensitivity $=6$ and Cultural Competence $=6$ ) was send back to the experts. The same methodology used in round 1 was also applied for round 2 .

Based on these a draft graphical representation of the model was done, including the agreed statements as to be discussed and piloted during the focus group.

\subsection{Focus group}

A focus group $(\mathrm{N}=7)$ with healthcare leaders, aiming to present and discuss the model, as to improve clarity of the statements and to verify their relevance, was conducted in Cyprus. The participants were nurse leaders from various clinical settings, community nursing practice and nursing education. Personal conduct by the research team and explanation about the research project was done. A focus group guide was developed based on the needs assessment and Delphi study and consisted of five parts: 1) Culturally aware and compassionate health care leadership; 2) Culturally knowledgeable and compassionate health care leadership; 3) Culturally sensitive and compassionate health care leadership 4) Culturally competent and compassionate health care leadership and 5) Experience and everyday practice. The participants singed a consent form before the initiation of the focus group. The local project team presented to the participants the purpose of the model, the methodology for its development and the draft model produced based on the findings. A discussion followed that lasted approximately 60 minutes. Data were recorded and transcribed. The analysis of the data focused on participants' understanding and subsequently the clarity of the content map of the model, and whether they agree on its relevance and importance to their everyday practice.

\subsection{Ethical considerations}

The study was approved by the National Bioethical Committee of Cyprus. Further, all principles of Belmont report were applied such as anonymity and confidentiality as well as the freedom of participation.

\section{Findings}

\subsection{Needs assessment survey}

One hundred and ninety-nine ( $\mathrm{N}=199)$ participants were involved (see Table 1). The vast majority of the sample was female ( $n=165,82.9 \%)$. Mean age of the participants is 43.6. More than $50 \%$ of the sample included head/leader nurses, senior nurses and faculty staff (leading positions) with the majority having more than five years of experience.

Table 1. Number of participants per country

\begin{tabular}{ll}
\hline Partner-Country & $\mathbf{N}(\%)$ \\
\hline UK & $48(24.1)$ \\
Turkey & $41(20.6)$ \\
Italy & $32(16.1)$ \\
Cyprus & $28(14.1)$ \\
Romania & $21(10.5)$ \\
Spain & $17(8.5)$ \\
Denmark & $12(6.1)$ \\
TOTAL & $199(100)$ \\
\hline
\end{tabular}

Regarding the element of the culturally competent and compassionate care in nursing, Cultural Sensitivity and Cultural Competence were scored highest as the most significant by the participants (see Figure 1). The leadership qualities needed by senior healthcare staff, the top five ranking were communicative, fair, compassionate, encouraging and organizational (see Figure 2).

The most frequently reported key elements (values and principles, knowledge and skills) needed of a CCC leader: Values and Principles: respect, equity, compassion, cultural competence, tolerance, humanity, professionalism; Knowledge: knowledge about different cultures, healthcare knowledge, communication skills; Skills: communication skills, empathy, experience, leadership skills, courage. 


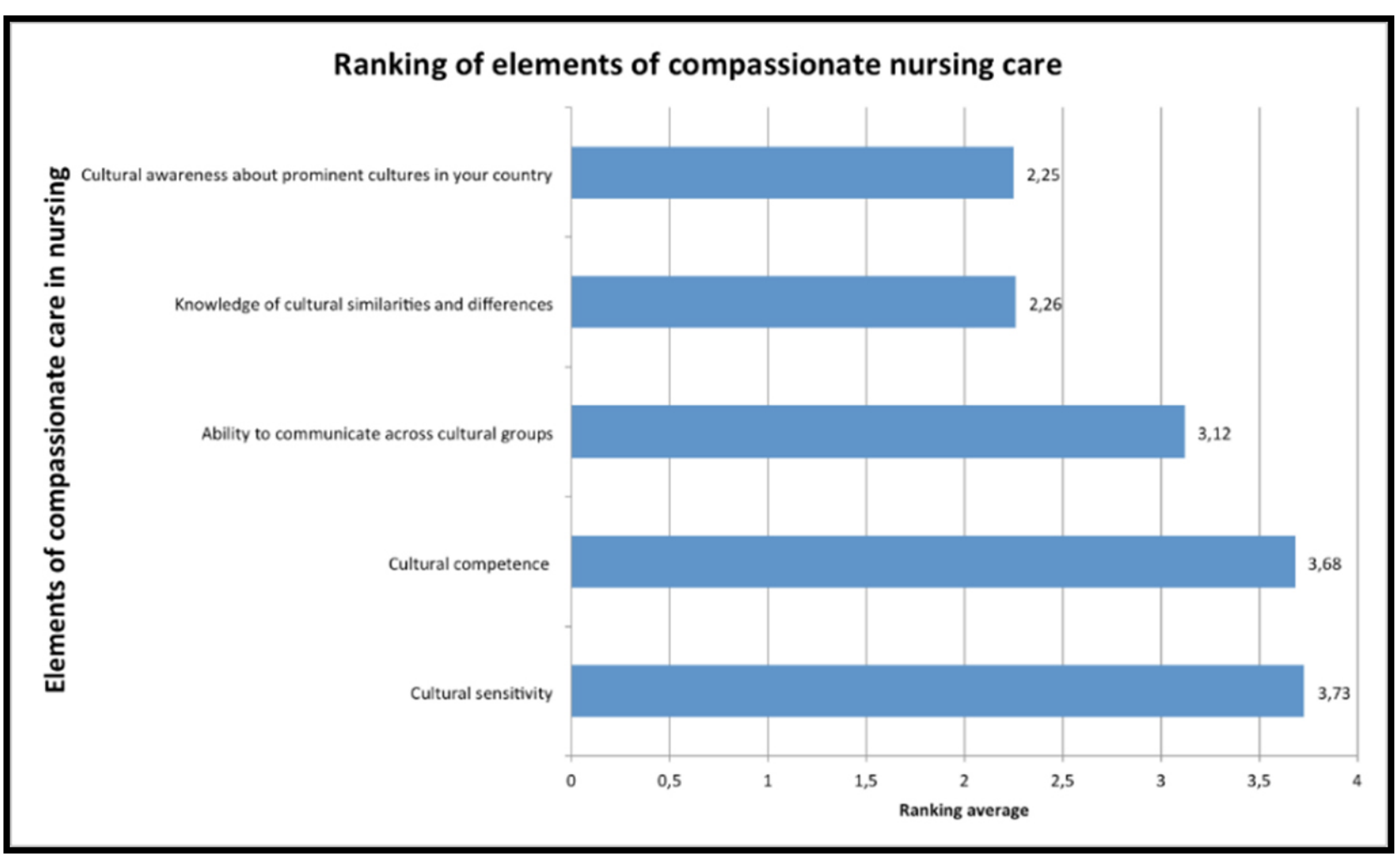

Figure 1. Elements of compassionate nursing care (rank 0-5)

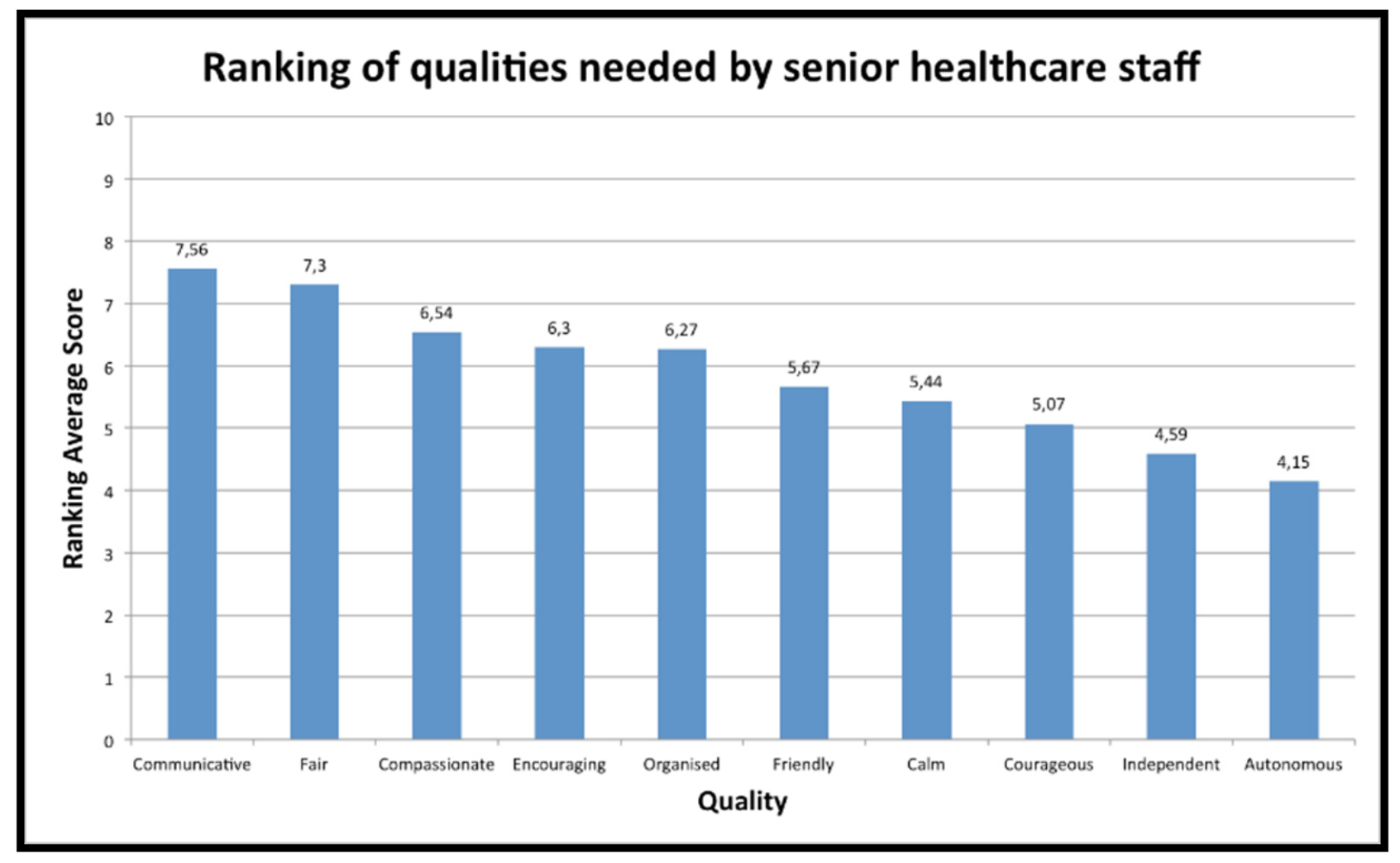

Figure 2. Key leadership qualities needed by senior healthcare staff as to promote a culturally competent and compassionate work culture (rank 0-10) 


\subsection{Delphi study}

After two rounds of Delphi study, it appeared that for the values-principles of 'tolerance', 'professionalism', 'communication skills' and 'encouraging' no related statements were retained. Thus, additional statements that were associated with these values-principles (4 statements in total-one for each value-principle) were developed and added. As mentioned above 41 indicating a consensus among the experts, and thus they were considered appropriate to be included in the formation of the model. Following these, the IENE team formulated the graphical representation of the model, showing the four stages, based on the Papadopoulos model ${ }^{[13]}$ and incorporated the agreed statements in each stage. This was done to ease understanding of the model from the health care professionals and leaders and apply it during the focus group, as it enables a smoother implementation of it.

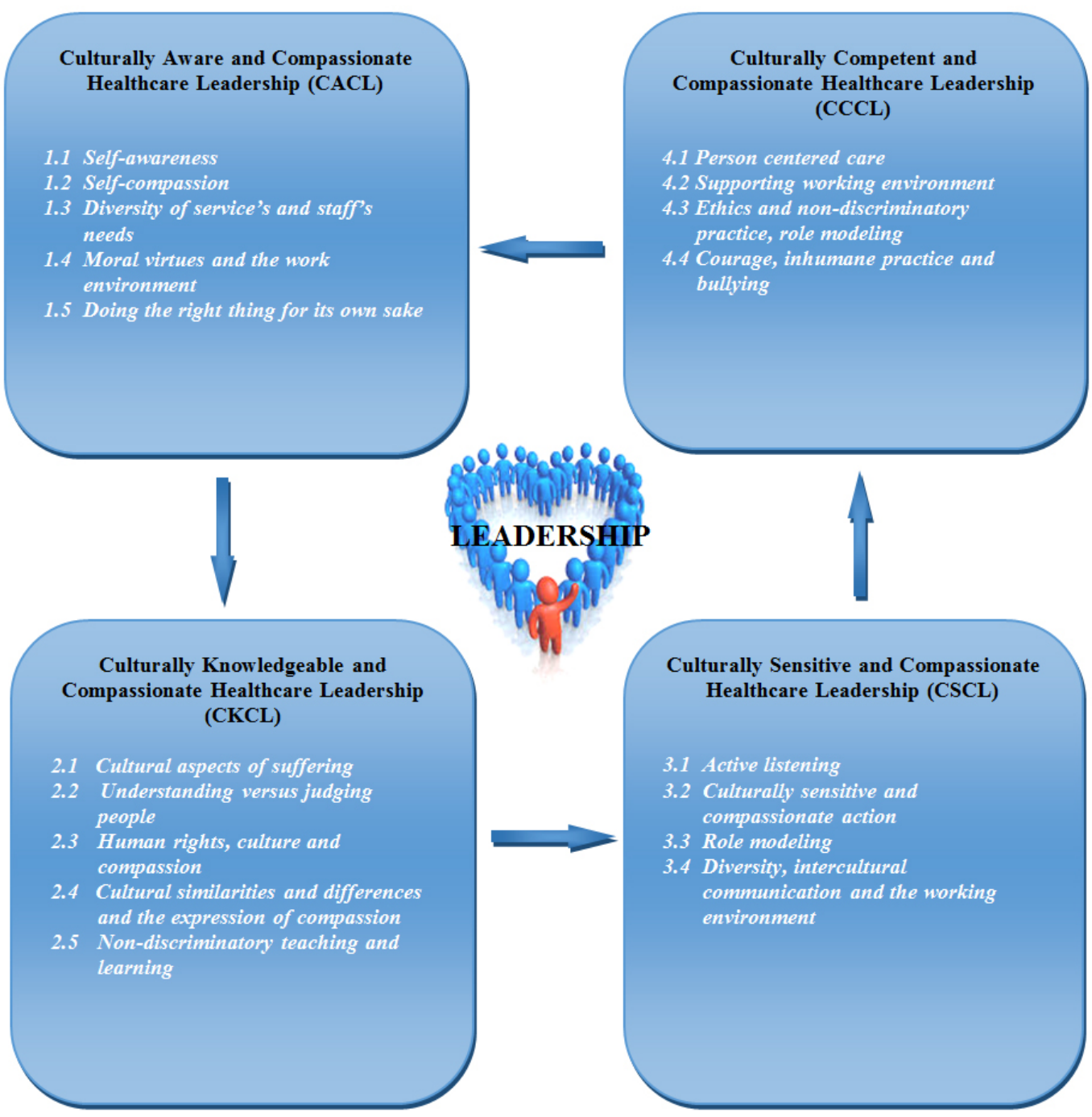

Figure 3. A European model for developing culturally competent and compassionate healthcare leadership

\subsection{Focus group}

Participants found the presentation of the project very informative and the project innovative. They stressed the great need for developing a model which would enable formal and informal leadership training to be focused around knowledge and skills which had not been addressed much before, on daily practice, such as cultural competence and compassion in leadership. Further, participants had some recommendations such as: Replacement of the word 'patients' with 'clients', as not all individual that use healthcare sector are patients (e.g. primary health care); point 1.5 (see Figure 3) not so clear to whom it refers to (e.g. leader for him/herself, leader and others, others only?); point 4.3 to emphasize role modeling, ethical issues- thus all items were rephrased. Overall, they found the model useful and relevant to current healthcare practices and that its underpinning philosophy and values, learning principles and suggested content map would contribute towards much needed culturally competent and 
compassionate healthcare services.

\section{Discussion}

In most contemporary European countries, health and social services are required to provide culturally competent healthcare. Cultural competence of healthcare staff was positively associated with the cultural competence of the leaders. This is however sensitive to contextual and individual factors. ${ }^{[15]}$ They further underlined that training for cultural competence should focus on developing skills such as kindness, empathy, openness and so on. ${ }^{[15]}$ These values are in line with the findings of the current study and included in the developed model.

The new NHS Leadership Model, emphasized autonomy, responsibility and accountability with a strong orientation towards patient care and compassion as this is the shift nowadays for the desired Leadership Model. ${ }^{[1]}$ This supports the equal emphasis that is given in the model of the current study on culturally competent and compassionate care.

In addition, another study argues that effective healthcare leadership includes several competencies. ${ }^{[2]}$ Among the highest rated ones are ethical values, effective communication, integrity and so on. These are also underlined in the Culturally Competent and Compassionate Health Care Leadership Model of this study. Further, where health service staff are well-led, this positively influences staff's satisfaction and patients' care, as they are treated with respect, care and compassion, ${ }^{[16]}$ values and competencies essential for a role model leader. It can be argued that having culturally competent leaders will enable an institution to become culturally competent. The benefits are social (e.g. increases trust, respect), health (e.g. increases preventative care, reduces care disparities) and business (e.g. improves efficiency) as well. ${ }^{[17]}$ Development and implementation of cultural competence strategies and related training require a reliance on leadership role-modeling within healthcare settings. Cultural competence of the leaders seemed to be associated with the cultural competence of the staff. ${ }^{[15]}$

Taking in consideration recent literature and based on the needs analysis, the Delphi study and focus group the main values of culturally competent and compassionate health care leader were revealed. These were distributed by the study consortium on the four stages of the Papadopoulos I. and Pezzella A., ${ }^{[18]}$ as they relate with the concept of leadership. This model incorporates most of the main values and competencies that have been found to be essential in other related studies and reports ${ }^{[1,2,15,16]}$ and goes beyond those combining cultural competence, compassion and leadership in health care.

Published by Sciedu Press

\section{The culturally competent and compassionate health care leadership model}

The model aims to provide the value, philosophy, educational principles and a conceptual map for potential content to aid trainers in developing curricula and educational tools for senior health care professionals who are considered important links in the development and sustainability of culturally sensitive and compassionate caring environments. Culturally competent and compassionate leadership is the synthesis and application of the four constructs used in this model (see Figure 3):

(1) Culturally aware and compassionate health care leadership

(2) Culturally knowledgeable and compassionate health care leadership

(3) Culturally sensitive and compassionate health care leadership

(4) Culturally competent and compassionate health care leadership

(1) Values, principles, knowledge and skills for a culturally aware and compassionate health care leadership (CACL)

1.1 Self-awareness as the first step for culturally competent compassionate leadership. Self-reflection in everyday practice is a necessary skill for all CCC leaders. Self-awareness will awaken the values of tolerance and acceptance for one's self and for others. Such awakening will enable the leader to understand the importance of the cultural identity of self and others.

1.2 Self-compassion is crucial for a culturally competent compassionate leader and will enable him/her to discharge his/her responsibilities and cope with all the stresses in the working environment. Kindness for one's self in combination with viewing one's own personal experiences as part of human condition are high qualities for a CCC leader.

1.3 Acknowledging the diversity of service patient'/users' and staff's needs should lead a CCC leader to treat them with compassion that is underpinned by respect, acceptance and caring of self and others.

1.4 Cultivating moral virtues in the work environment is characterized by a leader who inspires and motivates others by actions which demonstrate his/her respect in humanity and its diversity.

1.5 Doing the right thing for its own sake. CCC leaders cultivate practical wisdom in themselves and others in order to make the right decisions and take the right actions to benefit their service users and healthcare teams even in challenging situations. 
(2) Values, principles, knowledge and skills for a culturally knowledgeable and compassionate health care leadership (CKCL)

2.1 Acknowledging the cultural aspects of suffering indicates the unselfish/altruistic emotion, practised by a leader who understands that diversity may result in health inequality and exclusion. A culturally competent and compassionate leader adopts ethical practices.

2.2 Understanding rather than judging. When leaders value and practise equality, fairness and dignity, this will enhance loyalty and commitment of the staff during everyday practice. Adopting these as principles, a culturally competent and compassionate leader should strive to understand before s/he judges.

2.3 Deep understanding of human rights in relation to culture and compassion. Such understanding can maintain a positive equilibrium at workplace on the bases of equality and fairness. This will undoubtedly guide the leader to do the right thing for the right reasons.

2.4 Knowledge of cultural similarities and differences and their expression of compassion. Such knowledge is crucial for a CCC leader. Without it, s/he will be unable to build and deliver a safe and ethical healthcare service. S/he may risk alienating service users whilst at the same time not including essential elements of their individual differences which can be both an acknowledgement of their cultural identities and a useful resource of alternative care.

2.5 Non-discriminatory teaching and learning. Culturally competent and compassionate health care leaders should encourage and enable critical thinking, the adoption and promotion of reflective and participatory learning and teamwork skills. The leader should provide appropriate guidance and inclusive continuous support to their team.

(3) Values, principles and knowledge skills for a culturally sensitive and compassionate health care leadership (CSCL)

3.1 Active listening, dealing sensitively and culturally appropriately with others' feelings, needs, vulnerabilities and concerns are indicators of a culturally competent and compassionate leader. Active listening and open mindedness are crucial for a CCC leader.

3.2 Demonstrating culturally sensitive and compassionate actions enables the CCC leader to be a role model to the staff and set the standards to be followed and upheld when giving and receiving culturally competent compassion.

3.3 Role modelling. Culturally competent and compassionate healthcare leaders teach by examples and actions and promote learning through experience. Compassionate care is much more achievable where leaders show empathy and kindness.

3.4 Diversity, intercultural communication and the working environment. A culturally competent and compassionate leader creates a working environment that values diversity, practices and promotes intercultural communication and understanding.

(4) Values, principle and knowledge skills for a culturally competent and compassionate health care leadership (CCCL)

4.1 Person centred care. A culturally competent and compassionate healthcare leader should promote patient centred care on the bases of a culturally competent and compassionate needs assessment and care.

4.2 Supportive working environment. Such a working environment can be enabled by a leader who embeds and nurtures the values and principles of culturally competent and compassionate care. The healthcare working environment is frequently unpredictable, almost always complex and extremely busy. CCC leaders must possess the values skills, knowledge and flexibility in adapting to constantly changing conditions.

4.3 Ethics and non-discriminatory practice, role modelling. Learning to be a good role model requires a leader who aspires to be culturally competent and compassionate to acquire both theoretical knowhow and practical skills. Having the opportunity to practise being a role model under the guidance and support of a mentor will provide the foundations upon which s/he can build his/her style to add to his/her leadership. As alluded to previously, a leader cannot be CCC unless $\mathrm{s} /$ he respects the functions in ethical and non-discriminatory manner.

4.4 Being courageous in investigating and reporting cases of inhumane practice and bullying. Culturally competent and compassionate healthcare leaders must have the courage to take appropriate action and be able to speak out when are being witness or told about poor and inhumane practice to patients or bullying of staff and colleagues.

\section{Conclusion}

This model provides the theoretical and practical components needed for the development of culturally competent and compassionate healthcare leaders. The model can be used as a conceptual map for potential content to help health care trainers in the development of educational programs and educational tools for senior healthcare professionals, who are considered important links in the development and estab- 
lishment of culturally competent and compassionate caring healthcare environments.

Culturally competent and compassionate healthcare leaders are aware that can be influential and they are familiar in how to use it to improve care and implement their vision. Healthcare leaders understand that their influence can be critical in establishing collective intensity, passion and determination in achieving culturally competent and compassionate health care.

\section{ACKNOWLEDGEMENTS}

Authors would like to thank all partners that contributed to all stages of IENE 4 project.

\section{FUNDING}

This is part of a two-year EU Erasmus+ project.

\section{CONFLicts OF INTEREST Disclosure}

The authors declare that there is no conflict of interest.

\section{REFERENCES}

[1] Storey J, Holti R. Towards a new model for leadership for the NHS. UK: The Open University Business School 2013; [accessed 2019 March 13]. Available from: https://www. leadershipacademy. nhs.uk/wp-content/uploads/dlm_uploads/2014/10/Tow ards-a-New-Model-of-Leadership-2013.pdf

[2] Hargett CW, Doty JP, Hauck JN, et al. Developing a model for effective leadership in healthcare: A concept mapping approach. Journal of Healthcare Leadership. 2017; 9: 69-78. PMid:29355249 https://doi.org/10.2147/JHL.S141664

[3] West M, Armit K, Loewenthal L, et al. Leadership Development in Health Care: The Evidence Base. London: The Faculty of Medical Leadership and Management with The King's Fund and the Center for Creative Leadership. 2015; [accessed 2019 March 12] Available from: https://www.kingsfund.org.uk/sites/default/fil es/field/field_publication_file/leadership-leaders hip-development-health-care-feb-2015.pdf

[4] Klasko S. What healthcare will look like in 2020. TEDxPhiladelphia. 2014; [accessed 2018 November 5] Available from: https: //www . youtube. com/watch?v=esugL07XANg

[5] Garman AN, Brinkmeyer L, Gentry D, et al. Healthcare leadership 'outliers': An analysis of Senior administrators from the top U.S. hospitals. J Health Adm Educ. 2010; 27(2): 87-97.

[6] Al-Sawai A. Leadership of Healthcare Professionals: Where Do We Stand? Oman Medical Journal. 2013; 28(4): 285-287.

[7] Bramley L, Matiti M. How does it really feel to be in my shoes? Patients' experiences of compassion within nursing care and their perceptions of developing compassionate nurses. Journal of Clinical Nursing. 2014; 23(19-20): 2790-2799.

[8] Collins AS. Preventing Health Care-Associated Infections (Chapter 41) In Hughes, R. (ed). Patient Safety and Quality: An EvidenceBased Handbook for Nurses. Rockville (MD): Agency for Healthcare Research and Quality (US). 2008; [accessed 2019 March 16]; Available from: https://www.ncbi.nlm.nih.gov/books/NBK2683 /

[9] Smith S, James A, Brogan A, et al. Reflections about experiences of compassionate care from award winning undergraduate nurses-What, so what now what? Journal of Compassionate Health Care. 2016.

[10] Petersen R, Frederiksen L, Jansen MM, et al. IENE 4-Report on integrative literature reviews on: Universal components of compassion, Measuring culturally competent compassion, Learning
Culturally Competent Compassion in theory and practice. 2015. Available from: http://ieneproject.eu/download/Outputs/ Report $\% 20$ on $\% 20$ integrative $\% 20 \% 20$ reviews.pdf

[11] IENE 4 Project website. [accessed 2019 March 17]. Available from: http://www.ieneproject.eu

[12] Okoli C, Pawlowski SD. The Delphi method as a research tool: an example, design considerations and applications. Information \& Management. 2004; 42: 15-29. https://doi.org/10.1016/j.im.2 003.11 .002

[13] Papadopoulos I. Courage, Compassion and Cultural Competence. The 13th Anna Reynvaan Lecture, De Stadsschouwburg - Amsterdam City Theatre. Academic Medical Centre, University of Amsterdam, Netherlands. Unpublished keynote lecture. 2011; [accessed 2019 March 17] Available from: https://www.researchgate.net/publication/255978533 _Courage_Compassion_and_Cultural_Competence_The_13 th_Anna_Reynvaan_Lecture_19th_May_2011_De_Stadssch ouwburg_-_Amsterdam_City_Theatre_Academic_medical_ Centre_University_of_Amsterdam_Netherlands

[14] De Vet HCW, Terwee CB, Mokkink LB, et al. Measurement in Medicine: A Practical Guide. Cambridge University Press; 2011.

[15] Dauvrin M, Lorant V. Leadership and Cultural Competence of Healthcare Professionals: A Social Network Analysis. Nursing Research. 2015; 64(3): 200-210. PMid:25871625 https://doi.org/10.1 097/NNR.0000000000000092

[16] Dawson JF, West MA, Admasachew L, et al. NHS Staff Management and Health Service Quality: Results from the NHS Staff Survey and related data. London: Department of Health. 2011. Available from: https://assets.publishing.service.gov.uk/governmen t/uploads/system/uploads/attachment_data/file/2154 55/dh_129656.pdf

[17] Health Research \& Educational Trust. Becoming a culturally competent health care organization. Chicago, IL: Illinois. Health Research \& Educational Trust. 2013. [accessed 2019 March 18] Available from: http://www.diversityconnection.org/di versityconnection/membership/Resource $\% 20$ Center $\% 20 \mathrm{D}$ ocs/Equity $\% 20$ of $\% 20$ Care $\% 20$ Report $\% 20$ FINAL. pdf

[18] Papadopoulos I, Pezzella A. A snapshot review of culturally competent compassion as addressed in selected mental health textbooks for undergraduate nursing students. Journal of Compassionate Health Care. 2015. https://doi.org/10.1186/s40639-015-0012-5 